\title{
Multi-layered epigenetic regulation of IRS2 expression in the liver of obese individuals with type 2 diabetes
}

\author{
Christin Krause $^{1} \cdot$ Cathleen Geißler $^{1} \cdot$ Heidi Tackenberg ${ }^{1} \cdot$ Alexander T. El Gammal $^{2}$ - Stefan Wolter ${ }^{2}$. \\ Joachim Spranger ${ }^{3} \cdot$ Oliver Mann $^{2} \cdot$ Hendrik Lehnert ${ }^{1,4} \cdot$ Henriette Kirchner $^{1,4}(\mathbb{C}$
}

Received: 24 July 2019 / Accepted: 30 April 2020 / Published online: 24 July 2020

(C) The Author(s) 2020

\begin{abstract}
Aims/hypothesis IRS2 is an important molecular switch that mediates insulin signalling in the liver. IRS2 dysregulation is responsible for the phenomenon of selective insulin resistance that is observed in type 2 diabetes. We hypothesise that epigenetic mechanisms are involved in the regulation of IRS 2 in the liver of obese and type 2 diabetic individuals.

Methods DNA methylation of seven CpG sites was studied by bisulphite pyrosequencing and mRNA and microRNA (miRNA) expression was assessed by quantitative real-time PCR in liver biopsies of 50 obese non-diabetic and 31 obese type 2 diabetic participants, in a cross-sectional setting. Methylation-sensitive luciferase assays and electrophoretic mobility shift assays were performed. Furthermore, HepG2 cells were treated with insulin and high glucose concentrations to induce miRNA expression and IRS2 downregulation.

Results We found a significant downregulation of IRS2 expression in the liver of obese individuals with type 2 diabetes $(0.84 \pm$ 0.08 -fold change; $p=0.0833$; adjusted $p$ value $\left.\left[p_{\mathrm{a}}\right]=0.0417 ; n=31\right)$ in comparison with non-diabetic obese participants $(n=50)$. This downregulation correlated with hepatic IRS2 DNA methylation at CpG5. Additionally, CpG6, which is located in intron 1 of IRS2, was hypomethylated in type 2 diabetes; this site spans the sterol regulatory element binding transcription factor 1 (SREBF1) recognition motif, which likely acts as transcriptional repressor. The adjacent polymorphism rs4547213 (G>A) was significantly associated with DNA methylation at a specificity-protein-1 (SP1) binding site (CpG3). Moreover, DNA methylation of $\operatorname{cg} 25924746$, a CpG site located in the shore region of the IRS2 promoter-associated CpG island, was increased in the liver of individuals with type 2 diabetes, as compared with those without diabetes. A second epigenetic mechanism, upregulation of hepatic miRNA hsa-let-7e-5p (let-7e-5p) in obese individuals with type 2 diabetes $(n=29)$ vs non-diabetic obese individuals $(n=49)\left(1.2 \pm 0.08\right.$-fold change; $\left.p=0.0332 ; p_{\mathrm{a}}=0.0450\right)$, is likely to act synergistically with altered IRS2 DNA methylation to decrease IRS 2 expression. Mechanistic in vitro experiments demonstrated an acute upregulation of let-7e-5p expression and simultaneous IRS2 downregulation in a liver (HepG2) cell line upon hyperinsulinaemic and hyperglycaemic conditions.

Conclusions/interpretation Our study highlights a new multi-layered epigenetic network that could be involved in subtle dysregulation of IRS 2 in the liver of individuals with type 2 diabetes. This might lead to fine-tuning of IRS 2 expression and is likely to be supplementary to the already known factors regulating IRS 2 expression. Thereby, our findings could support the discovery of new diagnostic and therapeutic strategies for type 2 diabetes.
\end{abstract}

Keywords DNA methylation $\cdot$ Human liver $\cdot$ IRS2 $\cdot$ MicroRNA let7e $\cdot$ NAFLD $\cdot$ Type 2 diabetes

Electronic supplementary material The online version of this article (https://doi.org/10.1007/s00125-020-05212-6) contains peer-reviewed but unedited supplementary material, which is available to authorised users.

\section{Henriette Kirchner}

Henriette.Kirchner@uksh.de

Extended author information available on the last page of the article

$\begin{array}{ll}\text { Abbreviations } \\ \text { ALT } & \text { Alanine aminotransferase } \\ \text { CMV } & \text { Cytomegalovirus } \\ \text { EMSA } & \text { Electrophoretic mobility shift assay } \\ \text { eQTL } & \text { Expression quantitative trait loci } \\ \text { EWAS } & \text { Epigenome-wide association study } \\ \text { FDR } & \text { False discovery rate } \\ \text { FOXO } & \text { Forkhead box O }\end{array}$

Abbreviations Cytomegalovirus

Expression quantitative trait loci

EWAS Epigenome-wide association study

FOXO Forkhead box O 


\section{Research in context}

\section{What is already known about this subject?}

- Type 2 diabetes is a multifactorial disease that is influenced by genetic and non-genetic factors

- Epigenetic alterations in multiple tissues are associated with type 2 diabetes in humans and laboratory animal models

\section{What is the key question?}

- Are epigenetic alterations in the liver of obese individuals mechanistically involved in type 2 diabetes pathology?

\section{What are the new findings?}

- We show that IRS2 gene expression is downregulated in the liver of obese individuals with type 2 diabetes, which is partly influenced by altered IRS2 DNA methylation and the microRNA (miRNA) let-7e-5p

- IRS2 DNA methylation is associated with an intronic SNP that has been shown to be associated with type 2 diabetes in genome-wide association studies

- We show a novel multi-layered mechanism that might be involved in the regulation of hepatic IRS2 expression and that is influenced by epigenetic factors that are, in part, associated with genotype

\section{How might this impact on clinical practice in the foreseeable future?}

- Inhibiting hepatic miRNA let-7e-5p could potentially be useful in the future to enhance hepatic insulin signalling via IRS2 to ameliorate insulin resistance and hepatic steatosis

\section{GWAS Genome-wide association studies \\ IRE Insulin responsive element \\ MiRNA MicroRNA \\ NAFLD Non-alcoholic fatty liver disease \\ NAS Non-alcoholic fatty liver disease activity score \\ SP1 Specificity-protein-1 \\ SREBF1 Sterol regulatory element binding transcription factor 1 \\ UTR Untranslated region}

\section{Introduction}

IRS2 is one of the most important molecular switches mediating hepatic insulin receptor signalling and is involved in the phenomenon of selective insulin resistance, in which the lipogenic actions of insulin remain intact while glucose uptake and glycogen synthesis are disrupted $[1,2]$. Consequently, mice lacking Irs 2 exhibit selective insulin resistance [2] and specific knockout of Irs 2 in hepatocytes activates gluconeogenic pathways [3]. Moreover, leptin-deficient $o b / o b$ mice show a downregulation of Irs 2 expression simultaneously with increased expression of the gene encoding sterol regulatory element binding transcription factor 1 (Srebf1) [4]. Thus, we hypothesise that $I R S 2$ dysregulation plays a key role in the manifestation of type 2 diabetes, as well as liver steatosis, in humans. Previously it was shown that IRS2 expression is altered in obese individuals and in people suffering from non-alcoholic fatty liver disease (NAFLD) $[5,6]$; however, it currently remains unclear as to whether IRS2 contributes to the pathogenesis of type 2 diabetes and fatty liver disease in humans, and the mechanisms responsible for IRS2 dysregulation are unknown.

Epigenetic marks are regulated by environmental factors and their contribution to regulatory mechanisms of metabolic diseases is only starting to be unravelled. Aberrant expression of microRNAs (miRNAs) and changes in DNA methylation at $\mathrm{CpG}$ dinucleotides are responsible for the highly dynamic influence of environmental factors and lifestyle on gene expression, which can be passed on throughout generations [7]. The miRNA family hsa-let-7 is likely to regulate IRS2 expression and is associated with changes in glucose homeostasis in the muscle and brain $[8,9]$. DNA methylation at cytosines of $\mathrm{CpG}$ oligonucleotides influences gene expression, for example, by modulating the binding of transcription factors to the DNA [10]. Previously, a 3\% increase in IRS2 DNA methylation within a $\mathrm{CpG}$ island of exon $1(\operatorname{cg} 05514401)$ was identified in subcutaneous adipose tissue of 15 insulin-resistant obese women concomitant with decreased IRS2 gene expression, as compared with 14 non-obese normoglycaemic women [11], indicating that IRS 2 expression may be epigenetically regulated in metabolic diseases. Moreover, in a large-scale epigenomewide association study (EWAS), in blood samples, DNA methylation of the $\mathrm{CpG}$ site $\operatorname{cg} 25924746$, which is located in the shore region of the IRS2-related $\mathrm{CpG}$ island, was associated with type 2 diabetes [12]. However, functional consequences of these alterations in IRS2 DNA methylation remain unclear and whether they could lead to future glucose-lowering therapies is not yet known. 
Our study focuses uniquely on mechanisms leading to altered IRS2 expression in the liver of individuals with diabetes, as compared with non-diabetic participants, independent of classical risk factors, such as age, sex and obesity.

\section{Methods}

Study design and participants Liver wedge biopsies from segment III of the liver were obtained from 81 obese (BMI $>32 \mathrm{~kg} / \mathrm{m}^{2}$ ) male and female participants during bariatric surgery at the University Hospital Eppendorf (UKE; Hamburg, Germany), as previously described [13], in a cross-sectional study design. Blood samples for serum extraction were also drawn on the day of surgery, after an overnight fast. The study was approved by the local ethics committee of the Ärztekammer Hamburg (PV4889) and all participants gave informed consent (see electronic supplementary material [ESM] Methods).

Participants were categorised by clinical examination and use of glucose-lowering medication into type 2 diabetes $(n=$ $31)$ and non-diabetic control $(n=50)$ groups (ESM Table 1). All participants in the type 2 diabetes group were taking glucose-lowering medication. NAFLD activity score (NAS) was determined during surgery according to the current recommendations by two expert pathologists. For selected sub-analysis, this score was used for the stratification of individuals with $(\mathrm{NAS}=1-6)$ and without $(\mathrm{NAS}=0)$ liver steatosis and fibrosis. Clinical parameters were measured at the day of the surgery at the Institut für Klinische Chemie und Laboratoriumsmedizin, Zentrum für Diagnostik, Universitätsklinikum Eppendorf, Hamburg, Germany, according to the DIN EN ISO 15189:2014 certification. Glucose, cholesterol, HDL, aspartate aminotransferase (AST), alanine aminotransferase (ALT) and triacylglycerols were determined using photometric assays (kinetic bichromatic analysis; in-house assay by Institut für Klinische Chemie und Laboratoriumsmedizin, UKE, Germany). $\mathrm{HbA}_{1 \mathrm{c}}$ was quantified by capillary electrophoresis or by turbidimetric inhibition assays (in-house assay by Institut für Klinische Chemie und Laboratoriumsmedizin, UKE).

All samples were analysed in randomised order. Randomisation was achieved by processing the samples in the order of the biobank identification number, which was not in order or associated with the two study groups. Sample preparation and processing was blinded as all samples were only labelled with the biobank identification number. Data analysis was not blinded. Transcription-factor binding profiles were obtained from the JASPAR database [14].

DNA methylation measurement DNA was extracted from $25 \mathrm{mg}$ of snap frozen liver using the QIAmp mini kit (QIAGEN, Hilden, Germany). Genomic DNA was bisulphite-converted (bisDNA) using the EpiTectFast Bisulfite kit (QIAGEN). Bisulphite PCR was performed with the PyroMark PCR kit (QIAGEN). One primer pair was used to analyse $\mathrm{CpG}$ sites $1-5$, a second primer pair was used to analyse $\mathrm{CpG}$ site 6 and a third pair was used for analysis of cg25924746 (see ESM Table 2 for a list of primers, including primer for rs4547213). DNA methylation was measured by bisulphite pyrosequencing using PyroMark Q48 and PyroMark Q48 Advanced reagents (QIAGEN) (see ESM Methods).

RNA isolation and gene expression analysis Total RNA was extracted from $25 \mathrm{mg}$ of snap frozen liver using the Mirneasy mini kit (QIAGEN) and quantified spectrometrically. RNA $(2 \mu \mathrm{g})$ was reverse transcribed into cDNA using the SuperScript VILO cDNA synthesis kit (Invitrogen, Carlsbad, CA, USA).

Gene expression was measured in duplicates using TaqMan assays (Applied Biosystems, Foster City, CA, USA) and calculated with the $\Delta \Delta \mathrm{C}_{\mathrm{t}}$ method. The following genes were analysed: IRS2, PCK1, G6PC, PDK1, ACACA, ACACB, FASN, SCD, PNPLA2, FOXO1, ELOVL6. Gene expression was normalised to $C A S C 3$ expression (ESM Methods).

For hepatic miRNA cDNA synthesis, 10 ng of total RNA was reverse transcribed with the TaqMan Advanced miRNA cDNA synthesis kit (Applied Biosystems). Expression of hsalet-7e-5p (herein referred to as let-7e-5p) miRNA was measured in duplicates using TaqMan Advanced miRNA assays and calculated using the $\Delta \Delta \mathrm{C}_{\mathrm{t}}$ method, with hsamiR-24-3p being used as the housekeeping gene (ESM Methods).

Measurement of serum miRNA concentrations Serum miRNA was extracted using the recommended spike-in control celmiR-39-3p approach (miRNeasy Serum/Plasma Advanced kit; QIAGEN). MiRNAs were reverse transcribed with the qScript miRNA cDNA synthesis kit (QuantaBio, Beverly, MA, USA). Gene expression of let-7e-5p was measured by qPCR in duplicates using the FastStart Universal SYBR Green Master (Roche, Basel, Switzerland), self-designed qPCR primers (ESM Table 2) and a universal primer from the qScript kit. Expression was normalised to the spike-in control cel-miR-39-3p expression using the $\Delta \Delta C_{t}$ method (see ESM Methods).

Methylation-sensitive luciferase reporter gene assay Luciferase plasmids with a $\mathrm{CpG}$-free backbone and $\mathrm{CpG}-$ free cytomegalovirus (CMV) promoter (pCpGL-CMV-Fluc [15]) were created with different inserts (CpG1; rs4547213; CpG1-3; CpG1-6; rs4547213 and CpG2-6; ESM Table 2). Consequently, plasmids were either in vitro methylated using SssI methylase (NEB, Ipswich, MA, USA) or mock 
methylated (incubation without SssI). HepG2 cells were purchased from ATCC (Manassas, VA, USA) and were mycoplasma free. HepG2 were co-transfected with $100 \mathrm{ng}$ luciferase-reporter plasmid and either $500 \mathrm{ng} \beta$ galactosidase control plasmid (pCMV-bGal) or $10 \mathrm{ng}$ SV40 Renilla control plasmid (pRL-SV40) and luciferase activity was measured after $24 \mathrm{~h}$ of incubation (Promega, Madison, WI, USA). The luciferase assays were each performed three times, in triplicate (see ESM Methods).

Electrophoretic mobility shift assay Binding reactions containing HepG2 nuclear extracts (NE-PER Nuclear and Cytoplasmatic Extraction Reagents, ThermoFisher, Waltham, MA, USA) and biotinylated oligonucleotides were incubated prior to gel (Invitrogen) loading. For supershift assays, nuclear extracts were incubated with specific antibodies (polyclonal IgG $\alpha$ SREBF1 [PA1-46142] and polyclonal IgG $\alpha$ specificity-protein-1 [SP1; PA5-29165]; both Invitrogen). Protein-DNA complexes were plotted on nylon membranes and detected via chemiluminescence using the Chemiluminescent Nucleic Acid Detection Module (Thermo Fisher). The competitive erasure of protein binding to IRS2 was repeated five times to test for specificity of protein binding. Incubation with SREBF1 and SP1 antibodies was repeated three times. The electrophoretic mobility shift assay (EMSA) to determine the effect of SNP rs4547213 on protein binding was performed twice. One representative blot is shown (see ESM Methods).

Insulin treatment of HepG2 cells HepG2 cells were cultivated in high glucose (25 mmol/l) DMEM supplemented with $0.5 \%$ (wt/vol.) BSA and insulin (100 nmol/1 or $500 \mathrm{nmol} / 1$ Actrapid; NovoNordisk, Bagsværd, Denmark) or in low glucose (8.3 mmol/l) DMEM supplemented with 0.5\% (wt/vol.) BSA. After $24 \mathrm{~h}$ of treatment, miRNA-cDNA and mRNAcDNA synthesis steps were performed, as described above. The experiment was repeated three times and conducted in duplicates each time (see ESM Methods).

General statistics Data are expressed as means \pm SEM. Gene expression data was normalised to the respective control group. Results were either visualised as mean fold \pm SEM or normalised by $\mathrm{min} / \mathrm{max}$ normalisation to a scale from 0 (minimum expression) to 1 (maximum expression) for correlation analysis. Differences in DNA methylation were tested by twosided Wilcoxon rank-sum test and differences in gene expression by two-sided Student's $t$ test, or one-way ANOVA if more than two groups were compared, with a post hoc test to compare individual means. (GraphPad Prism, version 7; GraphPad Software, La Jolla, CA, USA). Post hoc testing was performed by comparing each mean value to the mean of the control condition. Post hoc-calculated $p$ values were adjusted for multiple testing by the false discovery rate
(FDR) method of Benjamini and Hochberg and a $q<0.05$ was assumed as significant. Spearman's correlation was used for ordinal values. For continuous variables, Pearson's correlation was calculated (MATLAB R2018a; The MathWorks, Natick, MA, USA). Significance was assumed at $p<0.05$ (see ESM Methods).

For association analysis between the polymorphism and the incidence of type 2 diabetes, a logistic regression analysis assuming age, sex and BMI as confounding factors was performed (MATLAB R2018a). Because age and sex was different between the non-diabetic and type 2-diabetic groups, multiple linear regression models using age, sex and BMI as cofactors were used to analyse gene expression and DNAmethylation data ( $\mathrm{R}$ version 3.5.1; RStudio, Boston, MA, USA). This is indicated by adjusted $p$ values $\left(p_{\mathrm{a}}\right)$ and all results that are $p_{\mathrm{a}}<0.05$ were considered significant independently of age, sex and BMI (ESM Table 3).

All data points were tested for outliers by the robust regression and outlier (ROUT) method in GraphPad Prism, version 7 and, subsequently, outliers were removed from the analysis. All correlation analyses were corrected for multiple testing using the Benjamini-Hochberg procedure, applying an FDR of $15 \%(q<0.15)$ (R version 3.5.1); $q$ values are provided in ESM Table 4 , and $p$ values and $q$ values are provided in ESM Table 5.

The effect of a polymorphism on IRS2 gene expression was assessed by an expression quantitative trait loci (eQTL) analysis, which was performed using a linear mixed-effect model in R, which included age, sex and BMI as cofactors for the calculation of the fitted model [16].

\section{Results}

Hepatic IRS2 expression is associated with liver health We measured hepatic IRS2 expression in liver tissue of obese individuals who were stratified by type 2 diabetes, as well as liver fibrosis and steatosis status (NAS). IRS2 expression was found to be decreased in the liver of obese individuals with type 2 diabetes $(n=31)$ vs obese individuals without type 2 diabetes $(n=50)\left(0.84 \pm 0.08\right.$-fold change; $p=0.0833 ; p_{\mathrm{a}}=$ 0.0417 ; Fig. 1a). IRS 2 expression correlated significantly with $\operatorname{NAS}\left(p_{\mathrm{a}}=0.0157 ; q=0.0722 ;\right.$ Fig. $\left.1 \mathrm{~b}\right)$ and serum $\operatorname{ALT}\left(p_{\mathrm{a}}=\right.$ $0.0143 ; q=0.0722$; Fig. 1c). Moreover, IRS2 expression correlated with $\mathrm{HbA}_{1 \mathrm{c}}\left(p_{\mathrm{a}}=0.0075 ; q=0.0578\right.$; Fig. 1d).

To investigate the impact of IRS2 dysregulation in the diabetic liver, we analysed expression of genes that are downstream of insulin signalling and involved in glucose and lipid metabolism. Hepatic IRS2 mRNA content directly correlated with gene expression of ACACA, FASN, SCD, PNPLA2, $P C K 1$ and $P D K 1$ (ESM Fig. 1a-j and ESM Table 5), indicating that a relationship exists between these genes. However, when stratified by groups, hepatic expression of these 
a
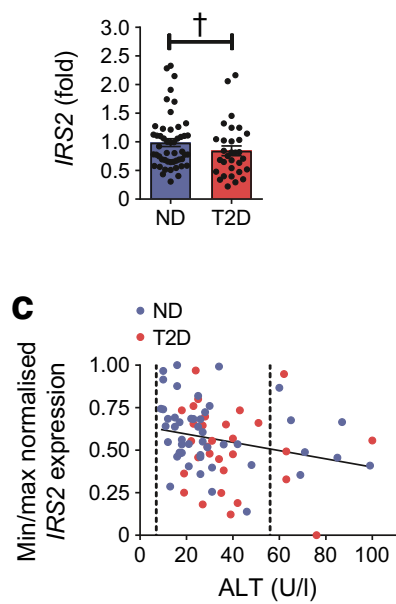
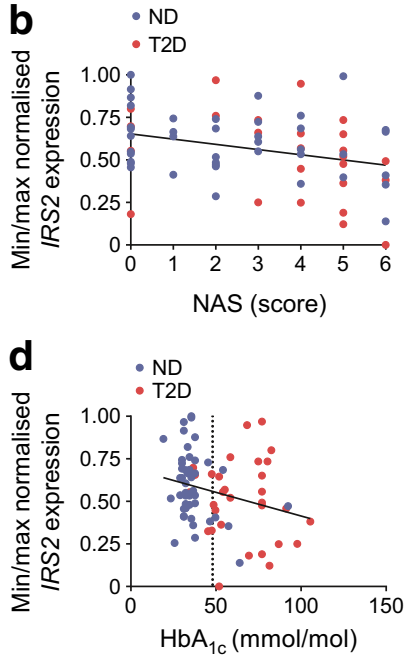

Fig. 1 The impact of hepatic IRS2 expression on liver health status. (a) Hepatic IRS2 expression is significantly decreased in individuals diagnosed with type 2 diabetes (T2D; $n=31$ ) in comparison with those without type 2 diabetes (ND; $n=50)$, independently of age, sex or BMI $\left(p_{\mathrm{a}}=\right.$ $0.0417)$. Gene expression was normalised to the expression of a housekeeper gene and is shown as mean fold \pm SEM relative to ND, as calculated by the $2^{-\Delta \Delta C_{t}}$ method. (b, c) IRS2 expression correlates with liver steatosis and fibrosis status (NAS) $\left(\mathbf{b} ; r=-0.2937, p_{\mathrm{a}}=0.0157 ; q=\right.$ $0.0722 ; n=68)$ and with serum concentration of ALT (c; $r=-0.2403$, $p_{\mathrm{a}}=0.0143 ; q=0.0722 ; n=78$ ). (d) Hepatic IRS2 expression correlates with $\mathrm{HbA}_{1 \mathrm{c}}$ values independently of age, sex and BMI $(r=-0.2668$, $\left.p_{\mathrm{a}}=0.0075 ; q=0.0578 ; n=68\right)$. In (c) and $(\mathbf{d})$, reference values are indicated by dashed lines. Pearson's correlation or Spearman's correlation $(p)$ and linear regression analysis $\left(p_{\mathrm{a}}\right)$ was used to calculate an association between gene expression $\left(\Delta \mathrm{C}_{\mathrm{t}}\right)$ and other parameters. For visualisation, gene expression was normalised by minimum $(\min ) /$ maximum $(\max )$ normalisation $\left(1-\left(x_{\mathrm{i}}-x_{\min }\right) /\left(x_{\max }-x_{\min }\right)\right)$, to a scale from 0 (min expression) to 1 (max expression), whereby $x_{\mathrm{i}}$ is the current $\Delta \mathrm{Ct}$ value of a specific gene, $x_{\min }$ is the minimum $\Delta \mathrm{Ct}$ value of all measurements of a specific gene and $x_{\max }$ is the maximum $\Delta \mathrm{Ct}$ value of all measurements of a specific gene. Gene expression is shown as normalised expression on the axes. $\uparrow p_{\mathrm{a}}<0.05$

downstream genes did not significantly change between obese type 2-diabetic and obese non-diabetic participants (ESM Fig. $1 \mathrm{k}, 1)$, except for $P D K 1$, which was increased in type 2 diabetes (ESM Fig. 11). Taken together, hepatic IRS2 is downregulated in the liver in those with diabetes and fatty liver, in comparison with the liver of obese non-diabetic participants. Importantly, our cohort showed negligible impact of confounding factors such as age, sex and BMI on IRS2 gene expression, as proven by linear regression models (Fig. 1) supporting our hypothesis that aberrant IRS2 expression might cause a diabetic phenotype independently of obesity.

\section{High variability of hepatic IRS2 DNA methylation within} transcription-factor binding motifs Because hepatic IRS2 expression is decreased in individuals with type 2 diabetes, we hypothesised that epigenetic mechanisms could play a role in this downregulation. Therefore, we performed in silico analysis for transcription-factor binding motifs containing a

CpG dinucleotide (Fig. 2a). Two transcription-factor motifs in proximity to each other (recognition sites for SP1 and SREBF1) were found in IRS2 intron 1 (Fig. 2b). Both transcription-factor binding motifs contain a central $\mathrm{CpG}$ site. Thus, we analysed DNA methylation of six CpG sites (CpG16) that span these SP1 and SREBF1 motifs in the liver of the obese non-diabetic and type 2 diabetic cohorts. Methylation of CpG6, which is centred in the SREBF1 E-box motif (Fig. 2b), was significantly decreased in the liver of individuals with type 2 diabetes $\left(-4.92 \% ; p=0.0152 ; p_{\mathrm{a}}=0.0029\right.$; Fig. $\left.2 \mathrm{c}\right)$. Additionally, we measured methylation at the IRS2 promoter-associated CpG site cg25924746 (Fig. 2b), which was recently associated with altered fasting insulin and fasting glucose in a blood-based epigenome-wide study [12]. DNA methylation of $\operatorname{cg} 25924746$ is sex-dependent ( $p=0.0004$ for test between sex; data not shown), and was found to increase in the liver of obese diabetic participants, as compared with non-diabetic participants $(4.83 \% ; p=0.0208$; Fig. 2d), and correlated with fasting glucose $\left(p_{\mathrm{a}}=0.0600 ; q=0.0652\right.$; Fig. 2f). CpG3 methylation, which is located in the SP1 GC-box motif (Fig. 2b), correlated significantly with $\mathrm{HbA}_{1 \mathrm{c}}\left(p_{\mathrm{a}}=\right.$ $0.0281 ; q=0.103$; Fig. 2e). Importantly, DNA methylation at CpG1-6 within intron 1 of IRS2 did not correlate with age, sex or BMI (data not shown).

Additionally, we assessed correlation of DNA methylation at the seven investigated $\mathrm{CpG}$ sites individually with IRS2 mRNA levels (Fig. 2g and ESM Fig. 2a-e [data for CpG1 not shown]). DNA methylation of $\mathrm{CpG} 5$ correlated with IRS2 expression in the liver of the entire cohort (Fig. $2 \mathrm{~g} ; r=$ $\left.0.3415, p_{\mathrm{a}}=0.0025 ; q=0.0283 ; n=81\right)$. DNA methylation of the other investigated $\mathrm{CpG}$ sites did not correlate with IRS2 gene expression after adjustment for age, sex and BMI, or after stratification for sex (data not shown), indicating that IRS2 DNA methylation may only have a subtle influence on IRS2 expression.

The SNP rs4547213 (G>A; also referred to as $\operatorname{cg} 12195446$, for example, in Illumina Infinium bead chip assay) affects the guanine of CpG1 [17]. Thus, DNA methylation at CpG1 is altered according to the genotype at this position and erased when individuals are homozygous carriers of the A allele (CpA-site; ESM Fig. 2f). Stratification for genotype did not result in significantly different DNA methylation at CpG1 (ESM Fig. 2f) between non-diabetic and type 2-diabetic obese participants. The $\mathrm{G}$ allele was numerically more frequent in individuals with type 2 diabetes in our cohort, but a $\chi^{2}$ test and regression analysis did not indicate a significant association between SNP rs4547213 and type 2 diabetes (ESM Fig. 2g). Surprisingly, the genotype at this SNP was significantly associated with DNA methylation at $\mathrm{CpG} 3\left(p=0.0234 ; p_{\mathrm{a}}=\right.$ 0.0348; ESM Fig. $2 \mathrm{~h}$ ) as the $\mathrm{G}$ allele was associated with lower $\mathrm{CpG} 3$ methylation in obese type 2 diabetic participants, as compared with those without diabetes $(p=0.0350$; ESM Fig. 2i). An eQTL analysis, including age, sex and BMI as 
a

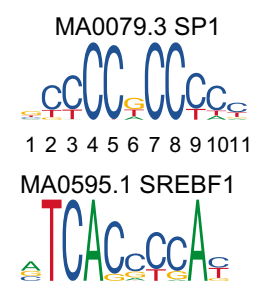

12345678910

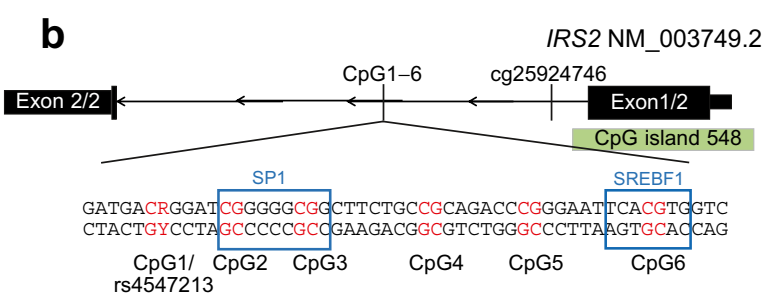

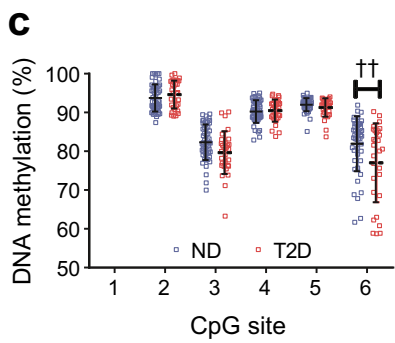
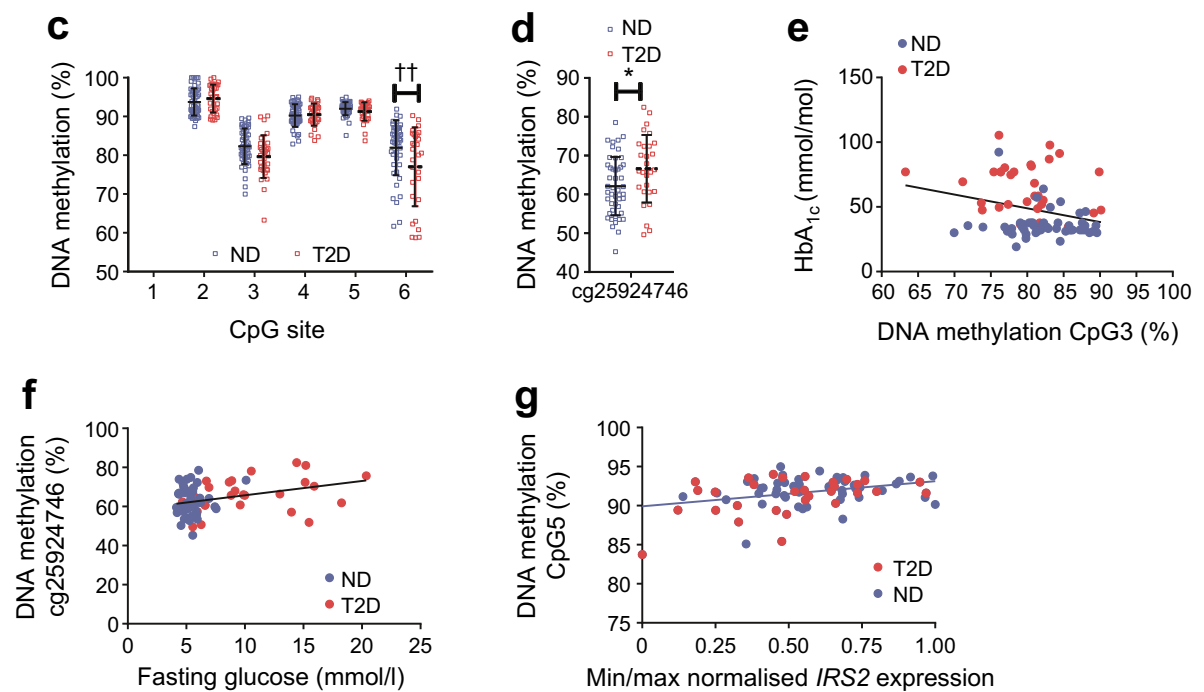

Fig. 2 DNA-methylation pattern and sequence snippet of intron 1 of IRS2. (a) Transcription-factor binding profiles of SP1 (GC-box) and SREBF1 (E-box) from JASPAR database [14]. (b) An overview of the IRS2 gene (NCBI reference sequence: NM_003749.2) and the location of the seven $\mathrm{CpG}$ sites analysed. The promoter-associated $\mathrm{CpG}$ island is shown in green. A sequence snippet from IRS2 intron 1, with the location of CpG1-CpG6 within a GC-box (SP1 binding) and E-box (SREBF1 binding) motif, is magnified. (c) The DNA-methylation pattern of CpG2-CpG6 after stratification by diabetes status (non-diabetic [ND], $n=50$; type 2 diabetic [T2D], $n=31$ ). CpG6 within the E-box motif shows a difference in DNA methylation of $4.92 \%$ between T2D and ND (linear regression analysis: $p=0.0152 ; p_{\mathrm{a}}=0.0029$ ). Each data point represents one individual; the black horizontal lines indicate the mean \pm SD. (d) The graph shows per cent hepatic DNA methylation at cg25924746 (CpG1; affected by rs4547213) in T2D and ND obese participants (ND, $n=44$; T2D, $n=28$ ). Methylation was significantly

cofactors, showed that the genotype at rs4547213 has no effect on IRS2 gene expression, per se ( $p>0.05$; ESM Fig. $2 \mathrm{j}$ ).

IRS2 DNA methylation alters reporter gene expression To test if DNA methylation at the six transcription factor-spanning CpG sites investigated within intron 1 of IRS2 has a functional effect on gene expression, we performed a methylationsensitive reporter gene assay [15]. DNA methylation reduces luciferase expression by about $50-75 \%$ when it is solely of CpG1 or the sequence spanning CpG1 to CpG6 and, therefore, the SP1/SREBF1 motifs were included in the reporter construct (Fig. 3a). The genotype at SNP rs4547213, in which $\mathrm{CpG1}$ is deleted, had no direct influence on luciferase expression, as tested by comparing the $0 \%$ methylated inserts

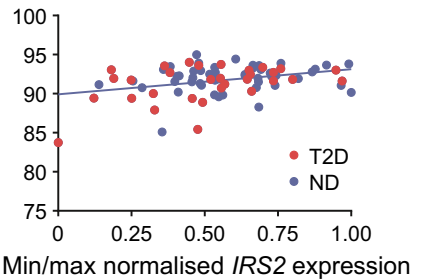

higher in T2D participants (mean difference, 4.83\%; $p=0.0208$ ). This finding was sex-dependent and diminished after adjustment for intracohort variability $\left(p_{\mathrm{a}}=0.0771\right)$. In $(\mathbf{c})$ and $(\mathbf{d})$, individual data points are shown with the mean (black horizontal line). (e) DNA methylation at CpG3 (GC-box) correlates negatively with $\mathrm{HbA}_{1 \mathrm{c}}$ values $(r=-0.2674$; $\left.p_{\mathrm{a}}=0.0281 ; q=0.103 ; n=81\right)$. (f) DNA methylation at $\operatorname{cg} 25924746$ correlates positively with fasting glucose $(r=0.3325, p=0.0043 ; n=$ 72). This finding was sex-dependent and diminished after adjustment for intracohort variability, and was not significant after correction for multiple testing ( $\left.p_{\mathrm{a}}=0.0600 ; q=0.3555\right)$. (g) DNA methylation of CpG5 correlates with IRS2 expression in the liver in the entire cohort $\left(r=0.3415, p_{\mathrm{a}}=0.0025 ; q=0.0283 ; n=81\right)$. Pearson's correlation $(p)$ and linear regression analysis $\left(p_{\mathrm{a}}\right)$ were used to calculate the association between DNA methylation and the investigated parameters. Max, maximum; min, minimum. $* p<0.05 ; \uparrow p_{\mathrm{a}}<0.01$

containing vectors harbouring the SNP with vectors containing the full $\mathrm{CpG}$ site 1 (Fig. 3b). Thus, the six CpGs identified that span the two transcription-factor binding sites appear to have a methylation-dependent regulatory effect on promoter activity.

Specific SP1 and SREBF1 binding to IRS2 According to public data the regions of IRS2 investigated here lie in a DNaseI hypersensitivity cluster [18], but transcription factor chromatin immunoprecipitation sequencing (ChIP-seq) peak data (Encyclopedia of DNA Elements [ENCODE3] project; [19, 20]) indicate no transcription factor binding in $\mathrm{HepG} 2$ cells or the liver. For confirmation of our in silico-proposed transcription-factor binding motifs, we performed an EMSA to test 

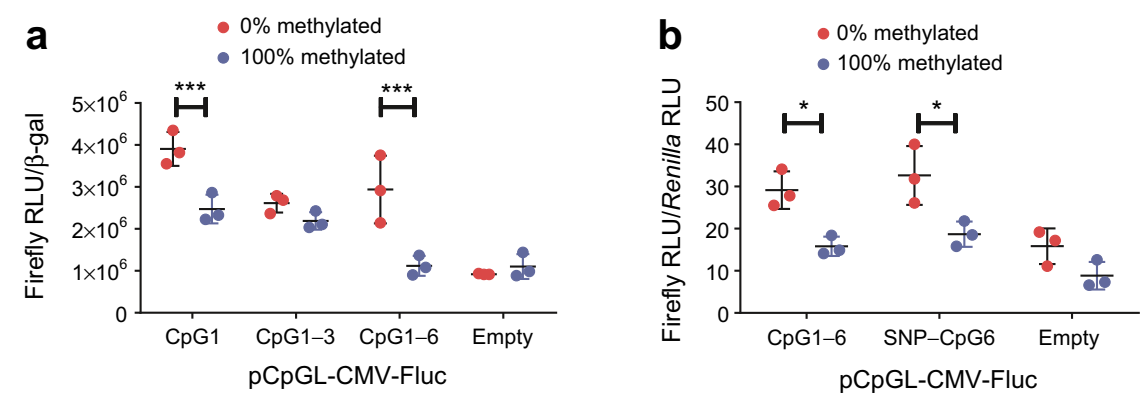

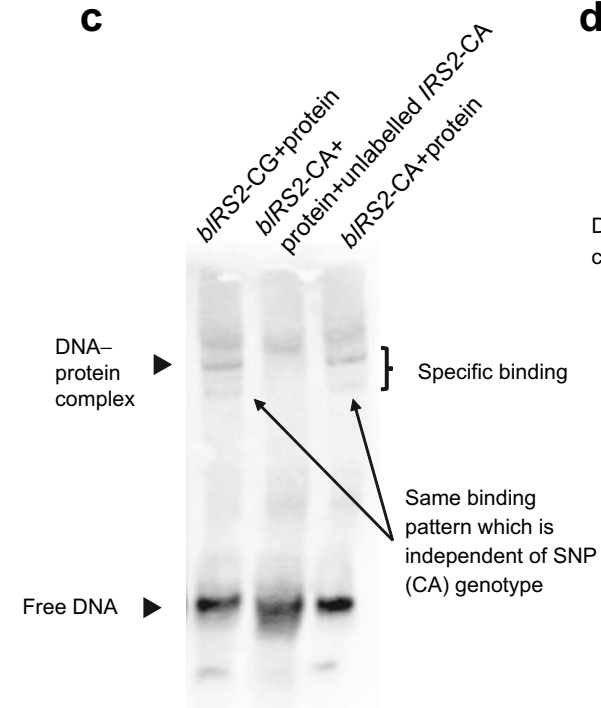

Fig. 3 Methylation-sensitive luciferase reporter gene assay and EMSA using HepG2 cells to prove mechanistic influence of $\mathrm{CpG}$ regions analysed and validation of specific binding of transcription factors. (a) A methylation-sensitive luciferase reporter gene assay shows significant upregulation of the Firefly luciferase gene for unmethylated plasmid DNA $(n=3)$. These results indicate that methylation solely at CpG1 or CpG1-CpG6 influences the CMV promoter of the Firefly-luciferaseencoding gene. (b) The methylation- and polymorphism-sensitive luciferase reporter gene assay shows no difference between luciferase expression with the SNP rs4547213 genotype (SNP-CpG6), in which CpG1 is deleted, and cells containing the full CpG-site 1 (CpG1-CpG6) or the SNP (SNP-CpG6) $(n=3)$. (c) Biotinylated DNA (bIRS2) oligonucleotides containing either the CG (lane 1) or CA (lanes 2 and 3) genotypes were incubated with HepG2 nuclear extracts (protein) for EMSA. The EMSA shows a shift of biotinylated DNA-protein complexes, meaning that protein was bound. In lane 2 , the reaction was additionally incubated with excess unlabelled DNA (unlabelled IRS2) which competes with the $\mathrm{b} I R S 2$ and specifically erases the shift of labelled DNA. There is no

whether nuclear protein would bind to our $\mathrm{CpG}$ sites of interest. Biotin-labelled oligonucleotides containing the six $\mathrm{CpG}$ sites (CpG1-6) were shifted, indicating that protein was bound (Fig. 3d). Incubation with nuclear extract from HepG2 cells led to a shift of labelled oligonucleotides (Fig. $3 \mathrm{c}, \mathrm{d})$. This shift was not influenced by the polymorphism rs4547213 (Fig. 3c). Incubation with different concentrations of unlabelled oligonucleotide led to a reduction of bound labelled oligonucleotide and, therefore, indicates specificity of binding (Fig. 3d). Incubation of nuclear extract with an antibody against SREBF1 reduced oligonucleotide-binding affinity, indicating that SREBF 1 can no longer bind the oligonucleotide when it is occupied by an antibody (Fig. 3d), whereas the SP1 antibody seemingly enhanced binding affinity. These results could indicate specific binding of the transcription factors SREBF1 and SP1 at their predicted motifs within intron 1 of IRS2.

Hepatic miRNA let-7e-5p is increased in type 2 diabetic participants Alongside DNA methylation, miRNAs represent 
another important epigenetic mechanism that regulates gene expression. Because epigenetic mechanisms often act synergistically, we performed data mining to identify miRNAs that possibly co-regulate IRS2 expression together with DNA methylation. Experimentally validated databases (DIsplacement ANAlyzer [DIANA] tools, TarBase v.8 [21]), as well at databases with predicted interactions (TargetScan Release 7.2 [22]), predict a conserved 8-mer site of let-7e-5p within the $3^{\prime}$ untranslated region (UTR) of IRS2. Hepatic let-7e-5p was increased $1.2 \pm 0.08$-fold in individuals with type 2 diabetes $(n=29)$, as compared with non-diabetic obese participants $(n=49)(p=$ 0.0332; $p_{\mathrm{a}}=0.0450 ;$ Fig. $4 \mathrm{a}$ ), and a negative correlation between let-7e-5p and IRS2 expression was found to exist $\left(r=-0.4133, p=1.7 \times 10^{-4} ; q=0.0019\right.$; Fig. 4b). Moreover, let-7e-5p expression was weakly correlated with NAS $(r=$ $0.2476 ; p_{\mathrm{a}}=0.0479 ; q=0.2123$; Fig. $\left.4 \mathrm{c}\right)$; this correlation was not significant after correction for multiple testing (ESM Table 4). Hepatic let-7e-5p expression did not correlate with age, sex or BMI (data not shown). To test whether let-7e-5p could be a candidate biomarker for type 2 diabetes in easily accessible tissues or body fluids, we measured let-7e-5p expression in non-haemolytic serum of $n=56$ participants. We did not find differences in serum let-7e-5p expression between type 2 diabetic and non-diabetic participants, and it correlated only weakly and not significantly with hepatic let-7e-5p expression after correction for multiple testing $(r=0.3284, p=0.0135 ; q=$ 0.2123; Fig. 4d, ESM Table 4).

Finally, we were interested to determine whether hepatic miRNA expression could be induced by hyperinsulinaemia and hyperglycaemia. We treated HepG2 cells with either $100 \mathrm{nmol} / \mathrm{l}$ or $500 \mathrm{nmol} / \mathrm{l}$ insulin in high-glucose medium, or with no insulin in low-glucose conditions as a control. HepG2 let-7e-5p expression was increased by acute hyperinsulinaemic and hyperglycaemic conditions as compared with the control (100 nmol/1 insulin: 2.4-fold; $500 \mathrm{nmol} / \mathrm{l}$ insulin: 2-fold; Fig. 4e). Concomitantly, gene expression of IRS2 was decreased and below the limit of detection in two out of three biological replicates in insulin-treated HepG2 cells (Fig. 4f). Because IRS2 expression after insulin treatment was only detectable in one of the three biological replicates, no statistical analysis could be performed.

\section{Discussion}

IRS2 is an essential mediator of insulin signalling [2]. Here, we show a multi-layered epigenetic mechanism that could be involved in downregulation of IRS2 expression in the liver of obese individuals with type 2 diabetes, as compared with obese individuals without type 2 diabetes. It is suggested that this regulation is facilitated by a combination of two epigenetic mechanisms, DNA methylation at SREBF1 and SP1 binding sites, and upregulation of hepatic miRNA let-7e-5p. DNA methylation of
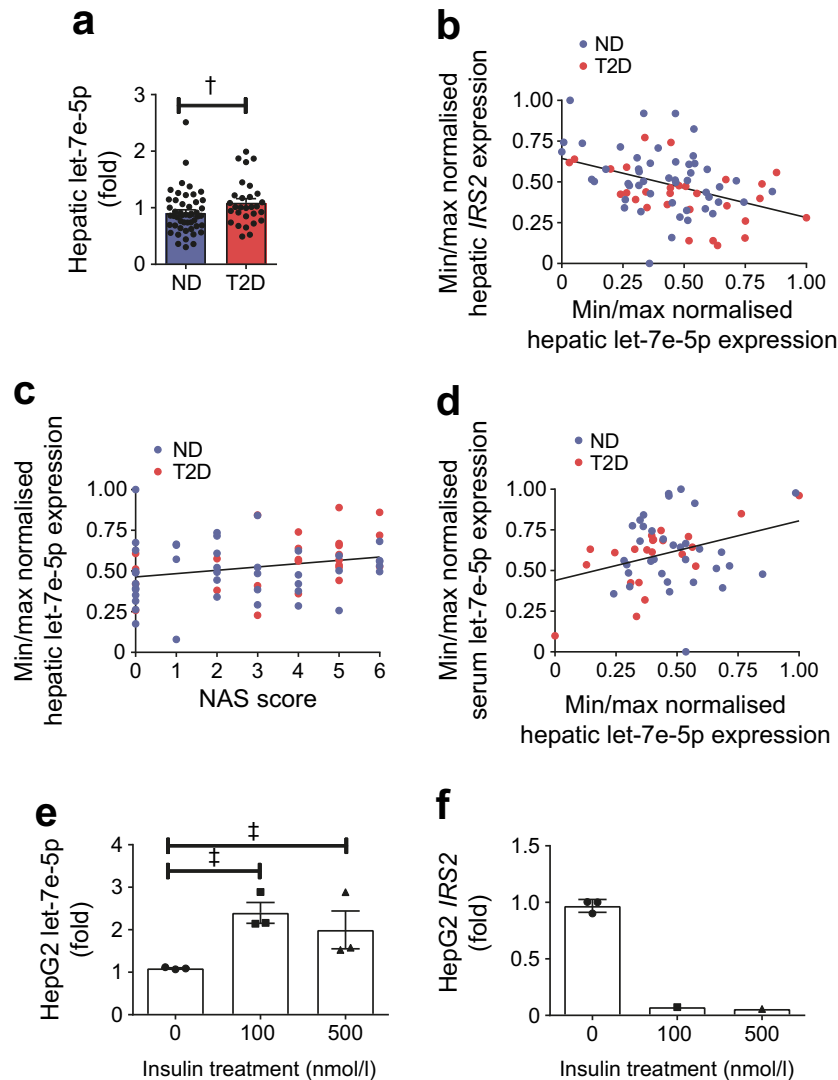

Fig. 4 Altered hepatic expression of miRNA let-7e-5p in type 2 diabetic (T2D) and non-diabetic (ND) participants. (a) Hepatic let-7e-5p expression is increased 1.2-fold in obese T2D individuals $\left(p_{\mathrm{a}}=0.0450 ; \mathrm{ND}, n=49\right.$; T2D, $n=29$ ). (b) Hepatic expression of IRS2 correlates negatively with let$7 \mathrm{e}-5 \mathrm{p}$ expression $\left(r=-0.4133, p=1.7 \times 10^{-4} ; q=0.0019 ; n=78\right)$. (c, d) Hepatic let-7e-5p expression does not correlate with liver steatosis and fibrosis status (NAS) (c; $\left.r=0.2476, p_{\mathrm{a}}=0.0479 ; \mathrm{q}=0.2123 ; n=67\right)$ or serum let-7e-5p expression $(\mathbf{d} ; r=0.3284, p=0.0135 ; q=0.2123 ; n=$ 56). (e) Intracellular expression of let-7e-5p is induced in HepG 2 cells after treatment with insulin $(100 \mathrm{nmol} / 1$ and $500 \mathrm{nmol} / \mathrm{l})$ in high-glucose medium $(25 \mathrm{mmol} / \mathrm{l})$ for $24 \mathrm{~h}$ analysed by one-way ANOVA $(p=0.0279$, overall difference between the means), with post-hoc test and adjustment for multiple testing by the FDR method $\left({ }^{\ddagger} q<0.05,0 \mathrm{nmol} / 1\right.$ vs $100 \mathrm{nmol} / 1$ or $0 \mathrm{nmol} /$ $1 \mathrm{vs} 500 \mathrm{nmol} / \mathrm{l}$ ); $n=3$. (f) Expression of IRS2 in HepG2 cells after treatment with insulin $(100 \mathrm{nmol} / 1$ and $500 \mathrm{nmol} / 1 ; n=3)$ in high-glucose medium $(25 \mathrm{mmol} / \mathrm{l})$ for $24 \mathrm{~h}$ is only detectable after insulin treatment for one experiment, therefore, no statistical analysis could be performed. MiRNA expression was normalised to the expression of a housekeeper miRNA (for liver and HepG2 cells) or to a spike-in control (for serum). In (a), (e) and (f), miRNA expression is shown as mean fold \pm SEM relative to ND (a) or $0 \mathrm{nmol} / 1$ insulin $(\mathbf{e}, \mathbf{f})$, as calculated by the $2^{-\Delta \Delta C_{t}}$ method. In (c) and (d), Pearson's correlation or Spearman's correlation $(p)$ and linear regression analysis $\left(p_{\mathrm{a}}\right)$ was used to calculate an association between gene expression $\left(\Delta C_{t}\right)$ and other parameters. For visualisation, miRNA expression was normalised by minimum $(\min ) /$ maximum $(\max )$ normalisation $\left(1-\left(\mathrm{x}_{\mathrm{i}}-\right.\right.$ $\left.\left.\mathrm{x}_{\min }\right) /\left(\mathrm{x}_{\max }-\mathrm{x}_{\min }\right)\right)$ to a scale from 0 (min expression) to 1 (max expression), whereby $x_{\mathrm{i}}$ is the current $\Delta \mathrm{Ct}$ value of a specific gene, $x_{\min }$ is the minimum $\Delta \mathrm{Ct}$ value of all measurements of a specific gene and $x_{\max }$ is the maximum $\Delta \mathrm{Ct}$ value of all measurements of a specific gene. Gene expression is shown as normalised expression on the axes. $\dagger p_{\mathrm{a}}<0.05 ;{ }^{\star} q<0.05$

the IRS2 promoter-associated EWAS marker cg25924746 is increased (in a sex-dependent manner) in the liver of type 2 


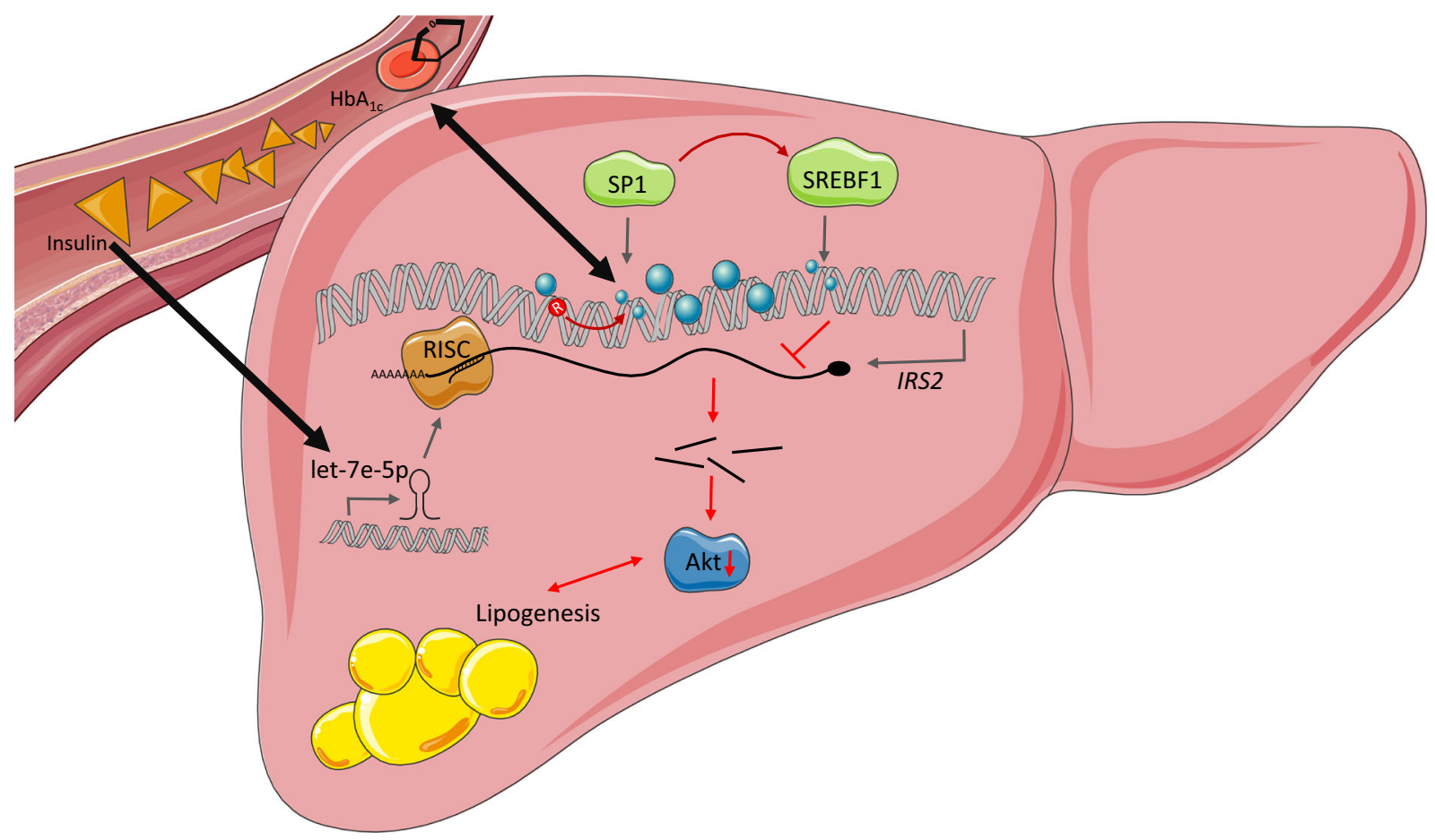

Fig. 5 Proposed multi-layered network participating in IRS2 dysregulation in type 2 diabetes. DNA methylation at SP1- and SREBF1-binding sites, together with elevated miRNA let-7e-5p levels, are associated with decreased IRS 2 transcription. Elevated let-7e-5p miRNA levels might result from increased serum insulin concentrations (single-ended thick black arrow) and might cause degradation of IRS2 mRNA (as illustrated by the short black lines above Akt), for example, via the RNA-induced silencing complex (RISC). A decrease in DNA methylation at the putative SP1 binding site is associated with increased $\mathrm{HbA}_{1 \mathrm{c}}$ levels (double-

diabetic participants, further strengthening our hypothesis that hepatic DNA methylation of IRS2 could play a role in type 2 diabetes. Additionally, the genotype at the intronic SNP rs4547213 is associated with DNA methylation at the SP1 binding site, which may, in part, explain why this SNP was associated with traits of glucose metabolism in a recent large-scale genomewide association study (GWAS) [23].

Basal regulation of IRS2 gene expression by transcription factors is well characterised. Forkhead transcription factors, such as forkhead box $\mathrm{O}$ (FOXO)1/3, bind to the insulin responsive element (IRE) of the IRS2 promoter to increase IRS2 expression during insulin stimulation, leading to an activation of the phosphatidylinositol 3-kinase (PI3K)/Akt1/2 signalling pathway [24]. The IRE of IRS2 overlaps with a sterol regulatory element (SRE), leading to competition between FOXO transcription factors and SREBF1a/1c, acting as a regulatory repressor of IRS2 [25]. Here, we propose additional regulators of hepatic IRS2 expression that might function as fine-tuning mechanisms depending on glycaemic state. One of the proposed transcription factors involved in this regulation is SREBF1. This factor is particularly interesting because it was previously shown to act as a translational repressor on IRS2 [25]. SREBF1c, the main SREBF1-isoform in the liver, is ended thick black arrow) and the genotype at rs4547213 (R, red arrow). This might lead to compromised insulin action, resulting in reduced Akt signal transmission and increased gluconeogenesis and lipogenesis, further fuelling liver steatosis (indicated as lipogenesis) and hepatic insulin resistance. This figure was created using images from Servier Medical Art (http://smart.servier.com). Servier Medical Art by Servier is licensed under a Creative Commons Attribution 3.0 Unported License

known to be involved in de novo lipogenesis by activating lipogenic genes, including fatty acid synthase (FASN) or stearyl CoA desaturase (SCD) [26, 27], which were found to be increased, although non-significantly, in our cohort (ESM Fig. $1 \mathrm{k}$ ), suggesting that SREBF1c is activated in our sample set. Consequently, through activation of SREBF1 and let-7e-5p, and the subsequent repression of IRS2 expression, the liver may be epigenetically arrested in a state of active lipogenesis.

SP1 is an important cofactor for the binding of SREBF1 to DNA [28]. Recently it was shown that DNA binding of SP1 $[29,30]$ and SREBF1 [31] is hindered by DNA methylation. In our study, methylation within the SREBF1 E-box motif was significantly decreased in individuals with type 2 diabetes, as compared with those without, despite receiving glucoselowering medication. Enhanced binding of SREBF1 due to DNA-hypomethylation might, therefore, be a mechanism that cannot be reversed by glucose-lowering medication and, instead, is putatively influenced by overall glycaemic control which is not as good in the type 2 diabetic group as compared with the non-diabetic group.

Because gene transcription can be regulated by DNA methylation of $\mathrm{CpG}$ islands in gene promoters, we studied DNA methylation of cg25924746. This CpG site is located in 
a CpG-island shore, which are generally known to have variable DNA methylation $[32,33]$. In our cohort, we reproduced the finding that DNA methylation of $\operatorname{cg} 25924746$ is altered in type 2 diabetes [12]. We found increased DNA methylation in the type 2 diabetic obese participants, as compared with non-diabetic obese subjects. However, correlations between cg25924746 and clinical parameters of glucose homeostasis were only significant in a sex-dependent manner, which became insignificant when adjusting for sex and for multiple testing. This discrepancy between our findings and previously published data could be due to the smaller sample size in our study and the fact that we lacked a lean control group. Furthermore, we investigated DNA methylation of cg25924746 in liver tissue, while it was previously only studied in DNA derived from leucocytes [12]. Therefore, caution should be applied when comparing DNA methylation across tissues [34].

The genotype at the neighbouring SNP rs4547213 correlates with DNA methylation at the $\mathrm{CpG} 3 / \mathrm{SP} 1$ motif. According to data in the GWAS central database [35], the polymorphism itself has been shown to be associated with fasting plasma glucose $(p=0.010$; as determined by the Meta-Analyses of Glucose and Insulin-related traits Consortium [MAGIC], which combined 21 GWAS) [36]. In addition, a second GWAS meta-analysis [23], which included more than 800,000 individuals of European ancestry from 32 GWAS, found an association between rs4547213 and the incidence of type 2 diabetes $\left(p=6.8 \times 10^{-7}\right)$. In our cohort, we could not reproduce the significant association between rs4547213 and type 2 diabetes, which is likely to be due to our smaller study cohort. Our mechanistic data from the luciferase reporter gene assay and the eQTL analysis did not show any effect of the polymorphism on gene expression per se. Nevertheless, type 2 diabetic participants with preserved DNA methylation due to the $\mathrm{G}$ allele at $\mathrm{CpG1}$, and not the putatively protective A allele, showed decreased DNA methylation at the $\mathrm{CpG} 3 / \mathrm{SP} 1$ motif in comparison with participants with type 2 diabetes and the AA genotype (ESM Fig. 2i). This could, in theory, lead to enhanced SP1/SREBF1 binding and downregulation of IRS 2 expression.

The findings from the luciferase reporter gene assay applied in this study indicates that higher methylation will result in decreased promoter activity. Our data derived from liver biopsies indicate a regulation in the opposite direction. It should be noted that the luciferase assay was used to analyse the effect of DNA methylation on a CMV promoter in an artificial cell-culture system and effects of competing transcription factors, as already described between SREBF1 and FOXO1/3, are not physiologically resembled. Nevertheless, we prove a mechanistic effect of DNA methylation on gene expression and the specificity of SREBF1 and SP1 binding, although our method was not sensitive enough to observe direct methylationdependent binding of both proteins.

Furthermore, hepatic let-7e-5p negatively correlated with IRS 2 expression in our cohort. Specific targeting of let-7e-5p to the 3'UTR of IRS2 has previously been established and has been specifically shown in the liver $[8,9,37,38]$. We, therefore, believe that let-7e-5p influences IRS 2 expression despite not finding correlations between hepatic let-7e-5p and clinical parameters after correction for multiple testing in our cohort. Moreover, high insulin and glucose treatment was able to acutely stimulate let-7e-5p expression in HepG 2 cells leading to IRS2 downregulation. A previous study on miRNAs of the let-7 family already pointed towards a function of let-7e-5p upregulation in disease progression of hepatocellular carcinoma (HCC), which is often a consequence of NAFLD [39]. Therefore, in the context of hepatic insulin resistance and fat accumulation, let-7e-5p could be an attractive target for miRNA inhibitor treatments, as seen for miRNA-122 in hepatitis C infection [40, 41].

Conclusion In conclusion, we identified a complex network of epigenetic DNA methylation, miRNA and genotypes that could be involved in the regulation of IRS 2 expression in the liver of diabetic individuals (Fig. 5). Within this network, our data suggest that let-7e-5p might play a larger role than DNA methylation in modulating hepatic IRS2 expression. As the targeting of miRNAs in liver is already used for human therapies [40, 41], inhibition of hepatic let-7e-5p might be a novel therapeutic target to support the treatment of hepatic insulin resistance and fatty liver.

Acknowledgements The authors thank M. Grohs (First Medical Department, University of Lübeck, Lübeck, Germany), N. Huckauf (Department of Endocrinology, Diabetes and Nutritional Medicine, Charité - Universitätsmedizin Berlin, Berlin, Germany), and P. Schroeder and A. Heinicke (both Department of General, Visceral and Thoracic Surgery, University Medical Center Hamburg-Eppendorf, Hamburg, Germany) for their excellent technical assistance.

Data availability The data that support the findings of this study are available from the corresponding author upon reasonable request.

Funding Open Access funding provided by Projekt DEAL. This work was supported by funding from the Deutsche Forschungsgemeinschaft (KI 1887/2-1 and GRK-1957).

Authors' relationships and activities The authors declare that there are no relationships or activities that might bias, or be perceived to bias, their work.

Contribution statement CK and HK designed experiments and assays, collected and analysed data and wrote the paper. CG and HT designed and performed bisulphite DNA experiments and analysed and interpreted 
data for critical revision. ATEG, SW, OM, JS and HL made a substantial contribution to the acquisition of clinical data and interpretation of results and revised the manuscript critically for important intellectual content. All authors read and approved the final manuscript. HK is the guarantor of this work and, as such, had full access to all the data in the study and takes responsibility for the integrity of the data and the accuracy of the data analysis.

Open Access This article is licensed under a Creative Commons Attribution 4.0 International License, which permits use, sharing, adaptation, distribution and reproduction in any medium or format, as long as you give appropriate credit to the original author(s) and the source, provide a link to the Creative Commons licence, and indicate if changes were made. The images or other third party material in this article are included in the article's Creative Commons licence, unless indicated otherwise in a credit line to the material. If material is not included in the article's Creative Commons licence and your intended use is not permitted by statutory regulation or exceeds the permitted use, you will need to obtain permission directly from the copyright holder. To view a copy of this licence, visit http://creativecommons.org/licenses/by/4.0/.

\section{References}

1. Brown MS, Goldstein JL (2008) Selective versus total insulin resistance: a pathogenic paradox. Cell Metab 7(2):95-96. https://doi. org/10.1016/j.cmet.2007.12.009

2. Kubota N, Kubota T, Kajiwara E et al (2016) Differential hepatic distribution of insulin receptor substrates causes selective insulin resistance in diabetes and obesity. Nat Commun 7:12977. https:// doi.org/10.1038/ncomms12977

3. Valverde AM, Burks DJ, Fabregat I et al (2003) Molecular mechanisms of insulin resistance in IRS-2-deficient hepatocytes. Diabetes 52(9):2239-2248. https://doi.org/10.2337/diabetes.52.9. 2239

4. Shimomura I, Matsuda M, Hammer RE, Bashmakov Y, Brown MS, Goldstein JL (2000) Decreased IRS-2 and increased SREBP1c lead to mixed insulin resistance and sensitivity in livers of lipodystrophic and ob/ob mice. Mol Cell 6(1):77-86. https://doi. org/10.1016/S1097-2765(05)00010-9

5. Rametta R, Mozzi E, Dongiovanni P et al (2013) Increased insulin receptor substrate 2 expression is associated with steatohepatitis and altered lipid metabolism in obese subjects. Int J Obes 37(7):986992. https://doi.org/10.1038/ijo.2012.181

6. Honma M, Sawada S, Ueno Y et al (2018) Selective insulin resistance with differential expressions of IRS-1 and IRS-2 in human NAFLD livers. Int J Obes 42(9):1544. https://doi.org/10.1038/ s41366-018-0062-9

7. Tomar AS, Tallapragada DSP, Nongmaithem SS, Shrestha S, Yajnik CS, Chandak GR (2015) Intrauterine programming of diabetes and adiposity. Curr Obes Rep 4(4):418-428. https://doi. org/10.1007/s13679-015-0175-6

8. Zhu H, Shyh-Chang N, Segrè AV et al (2011) The Lin28/let-7 axis regulates glucose metabolism. Cell 147(1):81-94. https://doi.org/ 10.1016/j.cell.2011.08.033

9. Boudreau RL, Jiang P, Gilmore BL et al (2014) Transcriptomewide discovery of microRNA binding sites in human brain. Neuron 81(2):294-305. https://doi.org/10.1016/j.neuron.2013.10. 062

10. Yin Y, Morgunova E, Jolma A et al (2017) Impact of cytosine methylation on DNA binding specificities of human transcription factors. Science 356(6337):eaaj2239. https://doi.org/10.1126/ science.aaj2239

11. Arner P, Sahlqvist A-S, Sinha I et al (2016) The epigenetic signature of systemic insulin resistance in obese women. Diabetologia 59(11):2393-2405. https://doi.org/10.1007/s00125-016-4074-5

12. Liu J, Carnero-Montoro E, van Dongen J et al (2019) An integrative cross-omics analysis of DNA methylation sites of glucose and insulin homeostasis. Nat Commun 10(1):2581. https://doi.org/10.1038/ s41467-019-10487-4

13. Krause C, Grohs M, El Gammal AT et al (2018) Reduced expression of thyroid hormone receptor $\beta$ in human nonalcoholic steatohepatitis. Endocr Connect 7(12):1448-1456. https://doi.org/ 10.1530/EC-18-0499

14. Khan A, Fornes O, Stigliani A et al (2018) JASPAR 2018: update of the open-access database of transcription factor binding profiles and its web framework. Nucleic Acids Res 46(D1):D260-D266. https://doi.org/10.1093/nar/gkx1126

15. Klug M, Rehli M (2006) Functional analysis of promoter $\mathrm{CpG}$ methylation using a $\mathrm{CpG}$-free luciferase reporter vector. Epigenetics 1(3):127-130. https://doi.org/10.4161/epi.1.3.3327

16. Strunz T, Grassmann F, Gayán J et al (2018) A mega-analysis of expression quantitative trait loci (eQTL) provides insight into the regulatory architecture of gene expression variation in liver. Sci Rep 8(1):1-11. https://doi.org/10.1038/s41598-018-24219-z

17. Bibikova M, Barnes B, Tsan C et al (2011) High density DNA methylation array with single $\mathrm{CpG}$ site resolution. Genomics 98(4):288-295. https://doi.org/10.1016/j.ygeno.2011.07.007

18. Thurman RE, Rynes E, Humbert R et al (2012) The accessible chromatin landscape of the human genome. Nature 489(7414): 75-82. https://doi.org/10.1038/nature11232

19. Wang J, Zhuang J, lyer S et al (2013) Factorbook.org: a Wiki-based database for transcription factor-binding data generated by the ENCODE Consortium. Nucleic Acids Res 41(D1):D171-D176. https://doi.org/10.1093/nar/gks1221

20. The ENCODE Project Consortium (2012) An integrated encyclopedia of DNA elements in the human genome. Nature 489(7414): 57-74. https://doi.org/10.1038/nature11247

21. Karagkouni D, Paraskevopoulou MD, Chatzopoulos S et al (2018) DIANA-TarBase v8: a decade-long collection of experimentally supported miRNA-gene interactions. Nucleic Acids Res 46(D1): D239-D245. https://doi.org/10.1093/nar/gkx1141

22. Agarwal V, Bell GW, Nam J-W, Bartel DP (2015) Predicting effective microRNA target sites in mammalian mRNAs. eLife 4:e05005. https://doi.org/10.7554/eLife.05005

23. Mahajan A, Taliun D, Thurner M et al (2018) Fine-mapping type 2 diabetes loci to single-variant resolution using high-density imputation and islet-specific epigenome maps. Nat Genet 50(11):1505. https://doi.org/10.1038/s41588-018-0241-6

24. Lu M, Wan M, Leavens KF et al (2012) Insulin regulates liver metabolism in vivo in the absence of hepatic Akt and Foxo1. Nat Med 18(3):388-395. https://doi.org/10.1038/nm.2686

25. Ide T, Shimano H, Yahagi N et al (2004) SREBPs suppress IRS-2mediated insulin signalling in the liver. Nat Cell Biol 6(4):351-357. https://doi.org/10.1038/ncb1111

26. Horton JD, Goldstein JL, Brown MS (2002) SREBPs: activators of the complete program of cholesterol and fatty acid synthesis in the liver. J Clin Invest 109(9):1125-1131. https://doi.org/10.1172/ JCI15593

27. Xu X, So J-S, Park J-G, Lee A-H (2013) Transcriptional control of hepatic lipid metabolism by SREBP and ChREBP. Semin Liver Dis 33(4):301-311. https://doi.org/10.1055/s-0033-1358523

28. Reed BD, Charos AE, Szekely AM, Weissman SM, Snyder M (2008) Genome-wide occupancy of SREBP1 and its partners NFY and SP1 reveals novel functional roles and combinatorial regulation of distinct classes of genes. PLoS Genet 4(7): e1000133. https://doi.org/10.1371/journal.pgen.1000133 
29. Zhu W-G, Srinivasan K, Dai Z et al (2003) Methylation of adjacent $\mathrm{CpG}$ sites affects $\mathrm{Sp} 1 / \mathrm{Sp} 3$ binding and activity in the p21Cip1 promoter. Mol Cell Biol 23(12):4056-4065. https://doi.org/10. 1128/MCB.23.12.4056-4065.2003

30. Douet V, Heller MB, Le Saux O (2007) DNA methylation and Sp1 binding determine the tissue-specific transcriptional activity of the mouse Abcc6 promoter. Biochem Biophys Res Commun 354(1): 66-71. https://doi.org/10.1016/j.bbrc.2006.12.151

31. Ehara T, Kamei Y, Takahashi M et al (2012) Role of DNA methylation in the regulation of lipogenic glycerol-3-phosphate acyltransferase 1 gene expression in the mouse neonatal liver. Diabetes 61(10):2442-2450. https://doi.org/10.2337/db11-1834

32. Irizarry RA, Ladd-Acosta C, Wen B et al (2009) The human colon cancer methylome shows similar hypo- and hypermethylation at conserved tissue-specific CpG island shores. Nat Genet 41(2): 178-186. https://doi.org/10.1038/ng.298

33. Hachiya T, Furukawa R, Shiwa Y et al (2017) Genome-wide identification of inter-individually variable DNA methylation sites improves the efficacy of epigenetic association studies. NPJ Genomic Med 2(1):11. https://doi.org/10.1038/s41525-017-0016-5

34. Krause C, Sievert H, Geißler C et al (2019) Critical evaluation of the DNA-methylation markers ABCG1 and SREBF1 for type 2 diabetes stratification. Epigenomics 11(8):885-897. https://doi. org/10.2217/epi-2018-0159

35. Beck T, Shorter T, Brookes AJ (2020) GWAS Central: a comprehensive resource for the discovery and comparison of genotype and phenotype data from genome-wide association studies. Nucleic Acids Res 48(D1):D933-D940. https://doi.org/10.1093/nar/gkz895
36. Dupuis J, Langenberg C, Prokopenko I et al (2010) New genetic loci implicated in fasting glucose homeostasis and their impact on type 2 diabetes risk. Nat Genet 42(2):105-116. https://doi.org/10. $1038 /$ ng. 520

37. Gao Y, Wu F, Zhou J et al (2014) The H19/let-7 double-negative feedback loop contributes to glucose metabolism in muscle cells. Nucleic Acids Res 42(22):13799-13811. https://doi.org/10.1093/ nar/gku1160

38. Frost RJA, Olson EN (2011) Control of glucose homeostasis and insulin sensitivity by the Let-7 family of microRNAs. Proc Natl Acad Sci 108(52):21075-21080. https://doi.org/10.1073/pnas. 1118922109

39. Shi W, Zhang Z, Yang B et al (2017) Overexpression of microRNA let-7 correlates with disease progression and poor prognosis in hepatocellular carcinoma. Medicine 96(32):e7764. https://doi.org/ 10.1097/MD.0000000000007764

40. van der Ree MH, van der Meer AJ, van Nuenen AC et al (2016) Miravirsen dosing in chronic hepatitis $\mathrm{C}$ patients results in decreased microRNA-122 levels without affecting other microRNAs in plasma. Aliment Pharmacol Ther 43(1):102-113. https://doi.org/10.1111/apt.13432

41. Jopling CL (2010) Targeting microRNA-122 to treat hepatitis C virus infection. Viruses 2(7):1382-1393. https://doi.org/10.3390/ v2071382

Publisher's note Springer Nature remains neutral with regard to jurisdictional claims in published maps and institutional affiliations.

\section{Affiliations}

\section{Christin Krause $^{1} \cdot$ Cathleen Geißler $^{1} \cdot$ Heidi Tackenberg ${ }^{1} \cdot$ Alexander T. El Gammal $^{2} \cdot$ Stefan Wolter ${ }^{2}$. Joachim Spranger ${ }^{3}$. Oliver Mann ${ }^{2} \cdot$ Hendrik Lehnert $^{1,4} \cdot$ Henriette Kirchner ${ }^{1,4}$}

1 First Department of Medicine, Division of Epigenetics and Metabolism, University of Lübeck, Ratzeburger Allee 160, 23562 Lübeck, Germany

2 Department of General, Visceral and Thoracic Surgery, University Medical Centre Hamburg-Eppendorf, Hamburg, Germany
3 Department of Endocrinology and Metabolism, Charité Universitätsmedizin Berlin, Berlin, Germany

4 German Center for Diabetes Research (DZD), MünchenNeuherberg, Germany 